\title{
Usefulness of Subtraction of 3D T2WI-DRIVE from Contrast- Enhanced 3D TIWI: Preoperative Evaluations of the Neurovascular Anatomy of Patients with Neurovascular Compression Syndrome
}

\author{
Y. Masuda, T. Yamamoto, H. Akutsu, M. Shiigai, T. Masumoto, E. Ishikawa, M. Matsuda, and A. Matsumura
}

\begin{abstract}
BACKGROUND AND PURPOSE: High-resolution 3D MR cisternography techniques such as 3D T2WI-driven equilibrium radiofrequency reset pulse (DRIVE) are used preoperatively to assess neurovascular anatomy in patients with neurovascular compression syndrome, but contrast between vessels and cranial nerves at the point of neurovascular contact is limited. The postprocessing technique subtraction of 3D T2WI-driven equilibrium radiofrequency reset pulse from contrast-enhanced 3D TIWI (sDRICE) provides both high spatial resolution and excellent contrast in depicting the neurovascular contact. We evaluated the usefulness of sDRICE compared with 3D T2WI-DRIVE.
\end{abstract}

MATERIALS AND METHODS: Twelve patients who underwent microvascular decompression for hemifacial spasm or trigeminal neuralgia were examined preoperatively with 3D T2WI-DRIVE and SDRICE. Two neuroradiologists retrospectively analyzed and scored lesion conspicuity, defined as the ease of discrimination between offending vessels and compressed nerves or the brain stem at the neurovascular contact. They also quantitatively analyzed the contrast and contrast-to-noise ratio at the neurovascular contact.

RESULTS: The lesion conspicuity scores of sDRICE images were significantly higher than those of 3D T2WI-DRIVE for all 12 patients $(P=.006)$ and the 6 cases of hemifacial spasm $(P=.023)$ but were not significantly higher in the 6 trigeminal neuralgia cases alone $(P=.102)$. For all 12 patients, the contrast-to-noise ratio between the offending vessels and the brain stem and between the vessels and nerves on sDRICE images was significantly higher than that on 3D T2WI-DRIVE $(P=.003$ and $P=.007$, respectively). Among these structures, the contrast values were also significantly higher on the sDRICE than on the 3D T2WI-DRIVE $(P<.001)$ images.

CONCLUSIONS: The postprocessing technique SDRICE is useful to evaluate neurovascular anatomy and to improve contrast and the contrast-to-noise ratio in patients with neurovascular compression syndrome.

ABBREVIATIONS: $\mathrm{CE}=$ contrast-enhanced; $\mathrm{CN}=$ cranial nerve; DRIVE $=$ driven equilibrium radiofrequency reset pulse; HFS = hemifacial spasm; $\mathrm{NVC}=$ neurovascular contact; sDRICE = subtraction of 3D T2WI-DRIVE from contrast-enhanced 3D TIWI; SI = signal intensity; TN $=$ trigeminal neuralgia

N eurovascular compression syndromes such as trigeminal neuralgia (TN) and hemifacial spasm (HFS) are characterized by hyperactive cranial nerve dysfunction. The most effective and standard treatment for this syndrome is microvascular decompression, which is capable of providing complete resolution of symptoms in most cases. ${ }^{1-4}$ A preoperative determination of microvascular anatomy in terms of the exact localization and direction of the compressing vessel and the anatomic relationship between cranial nerves and vessels is of great value to neurosurgeons. Some authors have proposed that the presence of clear-cut

Received May 29, 2014; accepted after revision August 5

From the Departments of Neurosurgery (Y.M., T.Y., H.A., E.I., M.M., A.M.) and Radiology (M.S., T.M.), Faculty of Medicine, University of Tsukuba, Tsukuba, Japan.

An earlier version of the article was previously presented at: Annual Meeting of the Japanese Society of Neuroradiology, February 24-26, 2011; Tokyo, Japan.

Please address correspondence to Hiroyoshi Akutsu, MD, 1-1-1 Tennodai, Tsukuba, Ibaraki 305-8575, Japan; e-mail: akutsuh@md.tsukuba.ac.jp

http://dx.doi.org/10.3174/ajnr.A4130 and marked vascular compression is a factor in good long-term prognosis ${ }^{5,6}$; thus, the accurate preoperative radiologic evaluation of the neurovascular anatomy is crucial to achieve an excellent clinical outcome. High-resolution 3D MR cisternography, such as 3D T2-weighted imaging driven equilibrium radiofrequency reset pulse (3D T2WI-DRIVE), ${ }^{7,8} 3$ F FIESTA $^{9,10}$ and 3D CISS, ${ }^{11-13}$ is the method of choice to evaluate preoperative neurovascular anatomy in patients with neurovascular compression syndrome.

Although these sequences have excellent spatial resolution, the contrast between vessels and cranial nerves at the point of neurovascular contact (NVC) is limited in general; this poor contrast is a major drawback of these sequences for preoperative evaluations of vascular compression syndromes. ${ }^{14,15}$ On 3 D TOF-MRA ${ }^{8,16-18}$ or 3D gadolinium-enhanced T1WI, ${ }^{8,13,18}$ vessels are clearly depicted but the nerves are too poorly seen to be depicted at the point of NVC. Fused images of 3D CTA or 3D TOF-MRA and 3D MR cisternography have been used to evaluate the NVC to 

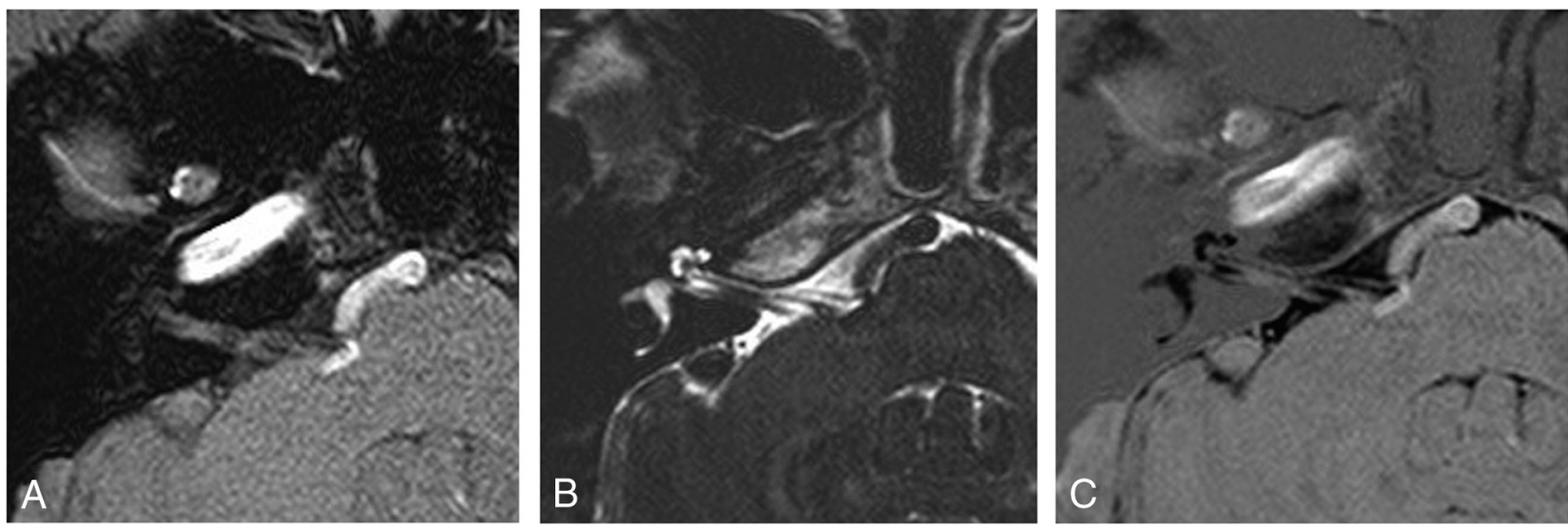

FIG 1. Method of image postprocessing. The first set of 3D T2WI-DRIVE source images (B) is subtracted from the second set of 3D CE TIWI (A) on a pixel-by-pixel basis by using workstations provided by the MR imaging system manufacturer. Then a third set of subtracted source images named sDRICE $(C)$ is produced.

solve this problem, ${ }^{19-23}$ but making these fusion images is technically challenging and too time-consuming for daily clinical practice.

We report a simple postprocessing technique that we named subtraction of 3D T2WI-DRIVE from contrast-enhanced 3D T1WI (sDRICE), which provides both high spatial resolution and excellent contrast between vessels and cranial nerves. It is easy and simple to create sDRICE images on a console without the need for any fusion technique or special software. We evaluated the utility of sDRICE imaging for the radiologic evaluation of patients with neurovascular compression syndrome, and we compared its efficacy with that of 3D T2WI-DRIVE, which is used in standard 3D MR cisternography.

\section{MATERIALS AND METHODS Study Population}

We studied 12 patients ( 9 women and 3 men; mean age, 53 years; range, 30-74 years) who underwent microvascular decompression for HFS or TN ( 6 cases of HFS and 6 cases of TN) at our institution between August 2009 and November 2013. All patients were examined with MR imaging by using both 3D T2WI-DRIVE and sDRICE before surgery. We retrospectively analyzed the patient data, including demographics, clinical history, and pre- and postoperative imaging findings. We analyzed the outcomes of the microvascular decompression in terms of cure and surgical complications on the basis of the criteria of the Japan Society for Microvascular Decompression Surgery. ${ }^{24}$ The final clinical outcome of microvascular decompression was evaluated 1 year after surgery.

\section{Image Acquisition}

MR imaging was performed by using a 3T whole-body MR imaging system (Achieva 3T; Philips Healthcare, Best, the Netherlands) with a manufacturer-provided 32-channel head coil for reception and a body coil for transmission. The MR imaging protocol was implemented by 1 author (T.M., with 20 years of experience in MR imaging). The MR imaging technique was as follows: axial 3D T2-weighted-DRIVE: TR, $2000 \mathrm{~ms}$; TE, $220 \mathrm{~ms}$; FOV, $160 \times 160 \mathrm{~mm}$; voxel resolution, $0.63 \times 0.63 \times 1.0 \mathrm{~mm}^{3}$; sensitivity encoding for fast MR imaging factor, 2; number of signals averaged, 1; echo-train length, 69; flip angle, $90^{\circ}$; acquisition time, 3 minutes 38 seconds. For the axial, contrast-enhanced 3D-T1-weighted fast-field echo images, we used the following parameters: TR, $30 \mathrm{~ms}$; TE, $4.1 \mathrm{~ms}$; FOV, $160 \times 160 \mathrm{~mm}$; voxel size, $0.63 \times 0.63 \times 1.0 \mathrm{~mm}^{3}$; sensitivity encoding for fast MR imaging factor, 2; number of signals averaged, 1 ; flip angle, $20^{\circ}$; acquisition time, 2 minutes 54 seconds. After a single intravenous Gd-DTPA (0.2 $\mathrm{mmol} / \mathrm{kg}$ ) injection was administered, a contrast-enhanced (CE) 3D T1-weighted sequence was run. We used 2 steps for simple and accurate subtraction after the acquisition: First, we matched the FOV and the voxel resolution between the 3D T2WI-DRIVE and CE 3D T1WI for the same scanning range. Second, to avoid misregistration, we shortened the interval time between the 2 sequences by scanning CE 3D T1WI in the train of 3D T2WI-DRIVE, and we asked the patients to stay still.

\section{Image Postprocessing}

After the data acquisition, the image data were transferred through a local area network for postprocessing by using the console software of the MR imaging system. The first set of 3D T2weighted-DRIVE source images (Fig $1 B$ ) was subtracted from the second set of CE 3D T1WI (Fig 1A) on a pixel-by-pixel basis by using workstations provided by the manufacturers of the MR imaging system. This process produced the third set of subtracted source images of sDRICE (Fig 1C). All of the source and subtracted images were sent to the PACS of the hospital. There was no rudimentary image registration technique before the image subtraction in our postprocessing program. To control the possibility of misregistration, we checked whether the diameters of cranial nerves (CNs) V and VII and those of the offending vessels on sDRICE were similar on 3D T2WI-DRIVE and CE 3D T1WI side by side. Two-dimensional axial, coronal, and sagittal fusion images were obtained to evaluate the neurovascular relationship in the cerebellopontine angle cistern, and if necessary, oblique images were reconstructed.

\section{Image Evaluation and Data Analysis}

We analyzed 3D T2WI-DRIVE and sDRICE both qualitatively and quantitatively. The qualitative image analysis was performed 
independently by 2 raters (T.M. and M.S. with 20 and 15 years of experience in neuroradiology, respectively) blinded to the hypothesis of the study and clinical data. The 3D T2WI-DRIVE and sDRICE images for each patient were independently evaluated in a random order of cases and sequences. Lesion conspicuity was defined as the ease of discrimination between offending vessels and compressed nerves or the brain stem at the NVC. To evaluate the lesion conspicuity, we used the following 3-point ordinal scoring system: 1, poor, not distinguishable due to no contrast; 2, good, distinguishable by slight contrast; and 3, excellent, easily distinguishable by clear contrast. The quantitative image analysis was performed by 2 authors (Y.M. and H.A).

The contrast-to-noise ratios (CNRs) were calculated on the basis of signal intensity (SI) and SD measurements in manually drawn ROIs. The ROIs were placed in the areas of the offending vessels and compressed parenchyma of the brain stem and com-

\section{Table 1: Patient data and outcomes}

\begin{tabular}{ccccccc}
\hline $\begin{array}{c}\text { Patient } \\
\text { No. }\end{array}$ & $\begin{array}{c}\text { Age }(\mathbf{y r}) / \\
\text { Sex }\end{array}$ & Disease & Side & $\begin{array}{c}\text { Offending } \\
\text { Vessel }\end{array}$ & Result Complication \\
\hline 1 & $46 / \mathrm{F}$ & HFS & R & AICA-PICA & Cure & No \\
2 & $30 / M$ & HFS & L & VA, AICA & Cure & No \\
3 & $53 / F$ & HFS & L & PICA & Cure & No \\
4 & $60 / M$ & HFS & L & VA, AICA & Cure & No \\
5 & $40 / F$ & HFS & L & PICA & Cure & No \\
6 & $50 / M$ & HFS & L & AICA & Cure & No \\
7 & $57 / F$ & TN & L & Petrosal vein & Cure & No \\
8 & $61 / F$ & TN & R & SCA & Cure & No \\
9 & $62 / F$ & TN & L & SCA & Cure & No \\
10 & $74 / F$ & TN & R & SCA & Cure & No \\
11 & $64 / F$ & TN & L & SCA, AICA & Cure & No \\
12 & $33 / F$ & TN & L & VA & Cure & No
\end{tabular}

Note:-AICA indicates anterior inferior cerebellar artery, PICA, posterior inferior cerebellar artery; SCA, superior cerebellar artery; VA, vertebral artery.
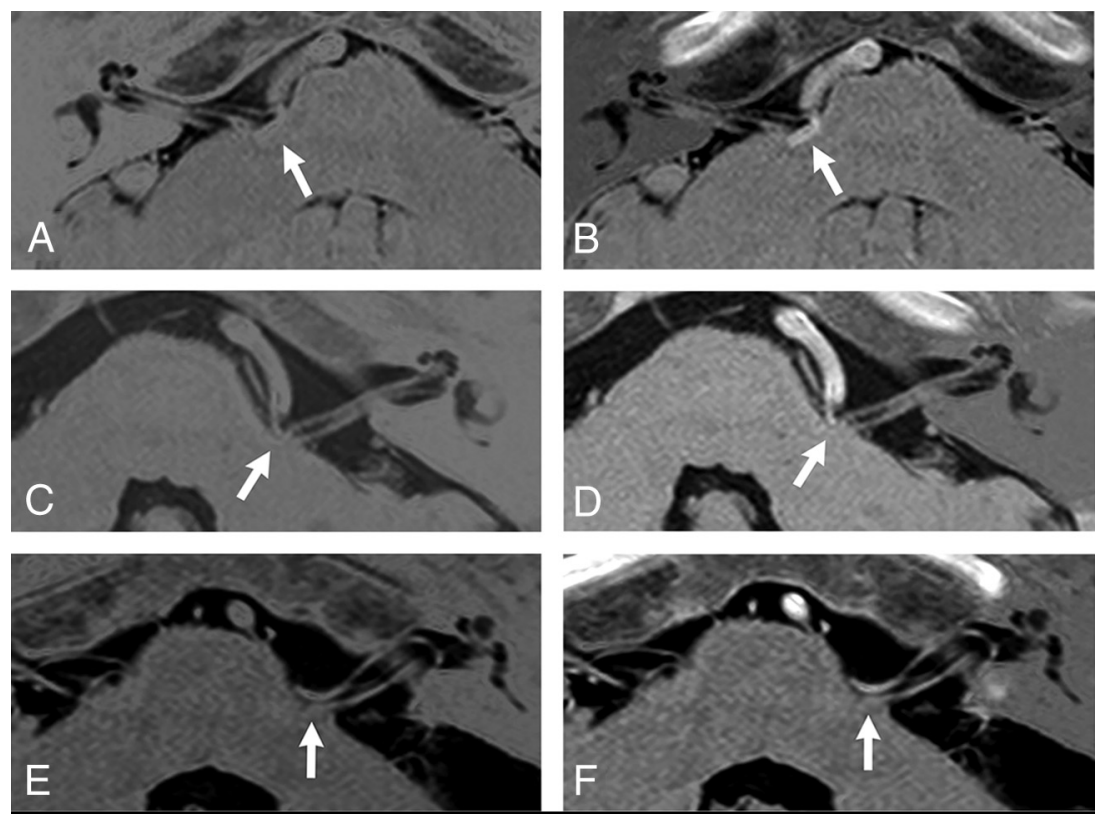

FIG 2. Comparison between the black/white-reversed 3D T2WI-DRIVE $(A, C, E)$ and sDRICE images $(B, D, F)$ at the NVC (arrow) in patients with HFS. In patients $1(A$ and $B)$ and $2(C$ and $D)$, the sDRICE images $(B$ and $D$ ) clearly show that the offending vessel invaginates into the brain stem and compresses the root exit zone of CN VII, in contrast to the 3D T2WI-DRIVE images ( $A$ and $C)$. In patient 6 ( $E$ and $F$ ), both images clearly show that the posterior cerebral artery compresses CN VII. pressed nerves at the NVC. Finally, the contrast and the CNRs of the offending vessels to brain stem and the offending vessels to compressed nerves were calculated by using the following equations: Contrast $t_{a / b}=2\left(S I_{\mathrm{a}}-S I_{\mathrm{b}}\right) /\left(S I_{\mathrm{a}}+S I_{\mathrm{b}}\right)$, and $C N R_{\mathrm{a} / \mathrm{b}}=$ $\left(S I_{\mathrm{a}}-S I_{\mathrm{b}}\right) / S D_{\mathrm{a}}$, where $\mathrm{a}$ and $\mathrm{b}$ denote the tissues of interest.

\section{Statistical Analysis}

A statistical analysis was performed by using standard statistical software (SPSS Version 15.0 for Microsoft Windows; IBM, Armonk, New York). An analysis of the interobserver agreement regarding qualitative variables was assessed by the $\kappa$ agreement test $(\kappa<0$, poor agreement; $0<\kappa<0.2$, slight agreement; $0.2<$ $<0.4$, fair agreement; $0.4<\kappa<0.6$, moderate agreement; $0.6<$ $\kappa<0.8$, substantial agreement; and $0.8<\kappa<1$, almost perfect (heement). ${ }^{25}$ Because there was substantial agreement between scores given by the 2 raters at $\kappa=0.660(P<.001)$, only the drom 1 rater (rater 1) were used for the statistical analstatistical significance of differences between 3D T2WIE and sDRICE was established by using the Wilcoxon signed the qualitative evaluation. The data in the quantitative

\section{RESULTS}

All of the imaging examinations by using both 3D T2-weighted-DRIVE and sDRICE were successfully completed before surgery. The clinical results are shown in Table 1. In all 12 patients, the offending vessels and compressing point predicted by the sDRICE images matched the intraoperative findings. In all patients, the preoperative HFS or TN was completely resolved after the microvascular decompression, and no complication was encountered.

sDRICE images and the black/whitereversed 3D T2WI-DRIVE images of patients with HFS and TN are shown in Figs 2 (HFS) and 3 (TN), respectively. The results of the lesion conspicuity scoring by the 2 raters are shown in Table 2. In all except 1 of the 6 patients with HFS, the lesion conspicuity in sDRICE was scored better than that in 3D T2WI-DRIVE by both raters. In particular, in patients 1 and 2 (Fig $2 A-D$ ), the sDRICE images were scored 2 points better than the $3 \mathrm{D}$ T2WI-DRIVE images by both raters. There was only 1 patient (patient 6 , Fig $2 E,-F$ ) for whom 1 rater scored the $3 \mathrm{D}$ T2WI-DRIVE and sDRICE images equally.

On the other hand, among the 6 patients with TN, there was only 1 patient for whom the sDRICE images were scored 2 points better than the 3D T2WI-DRIVE images by both raters (patient 7 , Fig $3 A$, $-B)$. In this patient, the offending vessel (the petrosal vein) was clearly depicted on the sDRICE images, in contrast to the 3D T2WI-DRIVE images. The 3D T2WI- 

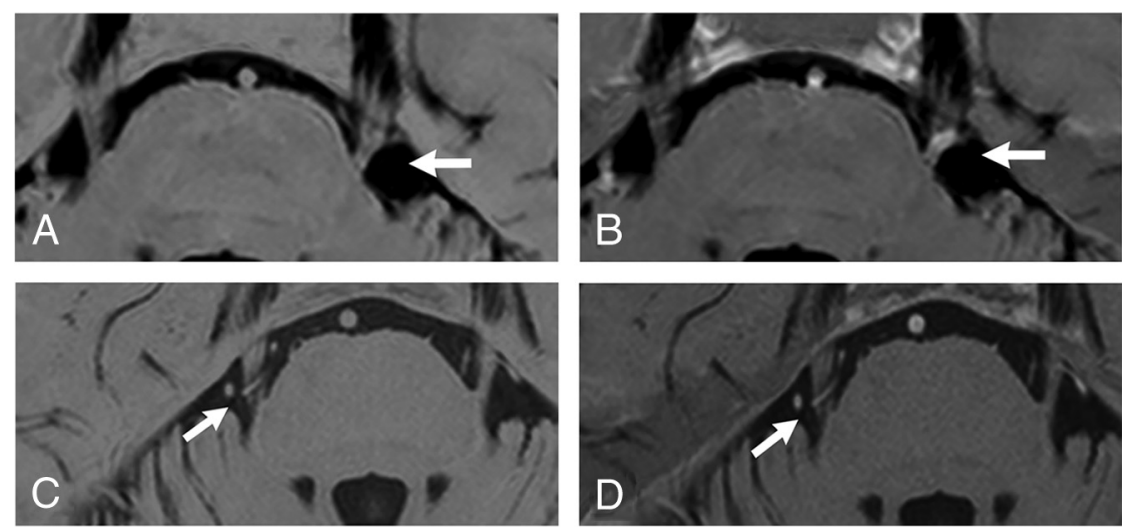

FIG 3. Comparison between the black/white-reversed 3D T2WI-DRIVE ( $A$ and $C$ ) and SDRICE images $(B$ and $D$ ) at the NVC (arrow) in patients with TN. In patient 7 ( $A$ and $B$ ), the sDRICE image $(B)$ clearly shows that the offending vessel (the petrosal vein) invaginates into $C N V$, in contrast to 3D T2WI-DRIVE $(A)$. In patient $10(C$ and $D)$, the offending vessel is relatively easily discriminated from CN V even on 3D T2WI-DRIVE $(D)$.

Table 2: The lesion conspicuity scoring by the 2 raters $^{a}$

\begin{tabular}{ccccccc}
\hline & & \multicolumn{2}{c}{ Scores by Rater 1 } & & \multicolumn{2}{c}{ Scores by Rater 2 } \\
\cline { 3 - 4 } \cline { 5 - 6 } $\begin{array}{c}\text { Patient } \\
\text { No. }\end{array}$ & Disease & T2WI-DRIVE & SDRICE & & $\begin{array}{c}\text { 3D } \\
\text { T2WI-DRIVE }\end{array}$ & SDRICE \\
\hline 1 & HFS & 1 & 3 & 1 & 3 \\
2 & HFS & 1 & 3 & 1 & 3 \\
3 & HFS & 1 & 2 & 1 & 3 \\
4 & HFS & 2 & 3 & & 2 & 3 \\
5 & HFS & 2 & 3 & & 2 & 3 \\
6 & HFS & 2 & 3 & & 2 \\
7 & TN & 1 & 3 & & 3 \\
8 & TN & 1 & 2 & & 3 \\
9 & TN & 2 & 3 & & 2 & 3 \\
10 & TN & 3 & 3 & & 2 & 3 \\
11 & TN & 3 & 3 & & 3 \\
12 & TN & 3 & 3 & 3 & 3 \\
\hline
\end{tabular}

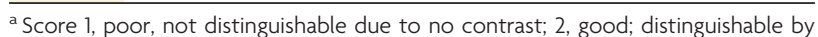
slight contrast; 3 , excellent, easily distinguishable by clear contrast.

DRIVE and sDRICE images were scored equally by rater 1 for patient 10 (Fig $3 C,-D$ ) and patient 11 and by both raters for patient 12. In those patients, the 3D T2WI-DRIVE and sDRICE images showed the offending vessel compressing CN V equally.

The results of the qualitative and quantitative analyses are summarized in Table 3. In the qualitative analysis, regarding the lesion conspicuity scored by rater 1 , the sDRICE images received significantly higher scores than the 3D T2WI-DRIVE images in all 12 patients $(P=.006)$ and in the patients with $\operatorname{HFS}(P=.023)$, but not in the patients with $\mathrm{TN}(P=.102)$.

In the quantitative analysis, the contrast values between the offending vessel and the brain stem and between the offending vessel and the compressed $\mathrm{CN}$ on sDRICE were significantly higher than those on 3D T2WI-DRIVE $(P<.001)$. This difference supports the results of the qualitative analysis for lesion conspicuity. The CNRs between the offending vessel and the brain stem and between the offending vessel and the compressed $\mathrm{CN}$ on sDRICE were also significantly higher than those on 3D T2WIDRIVE $(P=.003$ and $P=.007$, respectively).

\section{DISCUSSION}

MR cisternography performed with techniques such as 3D FIESTA, ${ }^{9,10} 3 \mathrm{D}$ CISS, ${ }^{11-13}$ 3D T2-weighted-DRIVE sequences, ${ }^{7,8}$ and reversed heavy $\mathrm{T} 2 \mathrm{WI}^{26}$ provides high spatial resolution and excellent visualization of vessels and cranial nerves and is thus a useful tool to depict cranial nerves and vessels in the cerebral cisterns. It is used for preoperative evaluations in cases of vascular compression syndrome, skull base tumors, and cerebrovascular disease. However, in these sequences, the contrast between vessels and cranial nerves is not always great enough, occasionally making a clear understanding of the anatomic structure difficult, especially in cases of neurovascular compression syndrome. ${ }^{9,14,15}$ Alternatives to standard MR cisternography (ie, image fusion between 3D CTA or 3D TOF-MRA and 3D MR cisternography) have been used to increase the contrast between vessels and cranial nerves. ${ }^{19-23}$ However, several disadvantages of such image fusion techniques have been reported. First, some data may be lost through the segmentation process so that a signal defect in vessels or nerves occurs. ${ }^{23}$ Second, artifacts are occasionally enhanced through the fusion process because artifacts of each technique are merged in the process. $^{20}$ Third, image fusion requires an extra software program and is time-consuming in most cases.

sDRICE can solve the above-mentioned limitations of the current standard MR cisternography because of its higher contrast without the use of any image fusion. In the present study, the lesion conspicuity of sDRICE was superior to that of 3D T2WIDRIVE. Unlike fusion images, sDRICE images can be easily and quickly created on a console, and sDRICE presents no risk of data loss during the subtraction process. In addition, artifacts from CSF flow or chemical shifts are somewhat reduced by the subtraction process in sDRICE. Considering these advantages, sDRICE may be applicable for other intracranial lesions such as skull base tumors adjacent to cranial nerves.

On the basis of the results of the present study, we found that sDRICE is more useful for understanding the anatomy at the NVC in patients with HFS than in those with TN. The reasons for this are as follows: In patients with HFS, because CN VII and the offending artery are almost the same in diameter, the discrimination of these structures is difficult on 3D T2WI-DRIVE. In addition, the compressing point is generally at the root exit zone of $\mathrm{CN}$ VII; thus, an offending artery tends to run adjacent to the brain stem, occasionally invaginating into the brain stem, which also makes it difficult to discriminate the artery from the brain stem due to the lower contrast (patient 1, Fig $2 A,-B$ ). In fact, in all except 1 of the 6 patients with HFS, the lesion conspicuity of sDRICE was scored better than that of 3D T2WI-DRIVE by both raters. There was only 1 patient with HFS (patient 6, Fig $2 E,-F$ ) for whom 1 rater scored the 3D T2WI-DRIVE and sDRICE images equally, and this outcome was because the offending artery was easy to discriminate from CN VII and the brain stem on both images. In this case, the existing thin CSF space between the offending artery and CN VII or brain stem may help in the discrimination of these structures. 
Table 3: Qualitative and quantitative analyses of 3D T2WI-DRIVE and SDRICE images

\begin{tabular}{|c|c|c|c|}
\hline Parameter & $\begin{array}{c}\text { 3D } \\
\text { T2WI-DRIVE }\end{array}$ & SDRICE & $\begin{array}{c}P \\
\text { Value }\end{array}$ \\
\hline \multicolumn{4}{|c|}{$\begin{array}{l}\text { Lesion conspicuity on the } \\
\text { neurovascular contact } \\
\text { point }^{\mathrm{a}}\end{array}$} \\
\hline \multicolumn{4}{|l|}{ Rater 1} \\
\hline Overall $(N=12)$ & $2.0(1-3)$ & $3.0(2-3)$ & .006 \\
\hline $\mathrm{HFS}(n=6)$ & $1.5(1-2)$ & $3.0(3-3)$ & .023 \\
\hline $\mathrm{TN}(n=6)$ & $2.5(1-3)$ & $3.0(2-3)$ & .102 \\
\hline \multicolumn{4}{|l|}{ Rater 2} \\
\hline Overall $(N=12)$ & $2.0(1-3)$ & $3.0(2-3)$ & .004 \\
\hline $\mathrm{HFS}(n=6)$ & $1.5(1-2)$ & $3.0(2-3)$ & .038 \\
\hline $\mathrm{TN}(n=6)$ & $2.0(1-3)$ & $3.0(3-3)$ & .038 \\
\hline \multicolumn{4}{|l|}{ Contrast $(N=12)^{b}$} \\
\hline Vessel/nerve & $-1.21 \pm 0.72$ & $0.28 \pm 0.15$ & $<.001$ \\
\hline Vessel/brain stem & $-1.08 \pm 0.67$ & $0.17 \pm 0.88$ & $<.001$ \\
\hline \multicolumn{4}{|l|}{$\operatorname{CNR}(N=12)^{\mathrm{b}}$} \\
\hline Vessel/nerve & $-1.80 \pm 1.47$ & $3.34 \pm 1.99$ & .007 \\
\hline Vessel/brain stem & $-0.97 \pm 0.58$ & $2.25 \pm 1.57$ & .003 \\
\hline
\end{tabular}

${ }^{a}$ Scoring data are listed for both raters as rater $1 /$ rater 2 and correspond to the median (with range) across patients. $P$ values were calculated with the Wilcoxon signed rank test.

${ }^{\mathrm{b}}$ Contrast and CNR data are means \pm SD. The $P$ values were calculated with a paired $t$ test. Contrast $t_{a / b}=2\left(S I_{a}-S I_{b}\right) /\left(S I_{a}+S I_{b}\right)$, and $C N R_{a / b}=\left(S I_{a}-S I_{b}\right) / S D_{a}$.

On the other hand, in the patients with TN, the offending artery (the superior cerebellar artery in most cases) was smaller in diameter than the trigeminal nerve, and it generally ran apart from the brain stem; thus, it was relatively easy to discriminate these structures, even on 3D T2WI-DRIVE without contrast enhancement. For the same reason, sDRICE may also be useful for cases of glossopharyngeal neuralgia, in which a cranial nerve and the offending vessel are similar in diameter.

As described in the "Materials and Methods" section, we used 2 steps for accurate subtraction without fusion to obtain the sDRICE images and controlled possible misregistration by using an internal check. First, we matched the FOV and the voxel resolution between 3D T2WI-DRIVE and CE 3D T1WI for the same scanning range. Second, we shortened the interval time between the 2 sequences by scanning CE 3D T1WI in the train of 3D T2WIDRIVE, and we asked the patients to keep still. In this study, because all patients were without cognitive deficits and were stable in physical status, subtraction without fusion was successfully completed in all of them. However, this method might have possible subtle misregistration; thus, we checked and confirmed whether the diameter of $\mathrm{CN} \mathrm{V}$ and VII and that of the offending vessels on sDRICE were similar on 3D T2WI-DRIVE and CE 3D T1WI side by side. Because of this possible misregistration, neuroradiologists separately evaluate 3D T2WI-DRIVE and CE 3D T1WI side by side for diagnostic purposes in clinical practice in our institution. Therefore, sDRICE images are used as adjuncts for more precise evaluation at neurovascular contact. On the other hand, for neurosurgeons, sDRICE has replaced the role of 3D T2-DRIVE and CE 3D T1WI for preoperative planning and intraoperative viewing.

Because sDRICE has excellent conspicuity at neurovascular contact and can depict both nerves and vessels on only 1 image set, it is more useful and practical for neurosurgeons than side-byside-displayed 3D T2WI-DRIVE and CE 3D T1WI. Only 1 disadvantage of sDRICE compared with the standard noncontrast MR cisternography was noted; with sDRICE, a contrast medium must be administered, which is contraindicated for patients with a history of allergy or renal dysfunction. However, an accurate preoperative evaluation of neurovascular anatomy is crucial for successful surgery in cases of neurovascular compression syndrome; thus, the use of contrast medium can be justified despite a possible-but-rare allergic reaction. In addition, contrast-enhanced images can depict not only arteries but also veins, in contrast to TOF imaging. ${ }^{12}$ Adjacent veins can be an offending vessel in cases of TN. Moreover, the identification of venous anatomy in the surgical field, especially of the petrosal vein or its collateral veins, is crucial to avoid postoperative venous infarction. Therefore, an enhanced MR image is always mandatory for the precise preoperative evaluation in cases of vascular compression syndrome.

\section{CONCLUSIONS}

sDRICE is a novel, simple, and useful postprocessing technique to evaluate neurovascular anatomy in patients with neurovascular compression syndrome. It is useful especially in patients with HFS for discriminating offending vessels from cranial nerves or the brain stem due to the excellent contrast between these structures.

Disclosures: Tetsuya Yamamoto_UNRELATED: Grants/Grants Pending: Grant-inAid for Society Collaboration from the Ministry of Education, Science and Culture, Japan, ${ }^{\star}$ Comments: topic on radiation therapy. Tomohiko Masumoto-UNRELATED: Consultancy: Bayer, Comments: blinded review reader for clinical trial. *Money paid to the institution.

\section{REFERENCES}

1. Jannetta PJ, Abbasy M, Maroon JC, et al. Etiology and definitive microsurgical treatment of hemifacial spasm: operative technique and results in 47 patients. J Neurosurg 1977;47:321-28

2. Barker FG 2nd, Jannetta PJ, Bissonette DJ, et al. Microvascular decompression for hemifacial spasm. J Neurosurg 1995;82:201-10

3. Barker FG 2nd, Jannetta PJ, Bissonette DJ, et al. The long-term outcome of microvascular decompression for trigeminal neuralgia. N Engl J Med 1996;334:1077-83

4. Samii M, Günther T, Iaconetta G, et al. Microvascular decompression to treat hemifacial spasm: long-term results for a consecutive series of 143 patients. Neurosurgery 2002;50:712-18

5. Sindou M, Leston J, Decullier E, et al. Microvascular decompression for primary trigeminal neuralgia: long-term effectiveness and prognostic factors in a series of 362 consecutive patients with clearcut neurovascular conflicts who underwent pure decompression. J Neurosurg 2007;107:1144-53

6. Zhang H, Lei D, You C, et al. The long-term outcome predictors of pure microvascular decompression for primary trigeminal neuralgia. World Neurosurg 2013;79:756-62

7. Ciftci E, Anik Y, Arslan A, et al. Driven equilibrium (drive) MR imaging of the cranial nerves V-VIII: comparison with the T2weighted 3D TSE sequence. Eur J Radiol 2004;51:234-40

8. Leal PR, Hermier M, Souza MA, et al. Visualization of vascular compression of the trigeminal nerve with high-resolution 3T MRI: a prospective study comparing preoperative imaging analysis to surgical findings in $\mathbf{4 0}$ consecutive patients who underwent microvascular decompression for trigeminal neuralgia. Neurosurgery 2011; 69:15-25

9. Benes L, Shiratori K, Gurschi M, et al. Is preoperative high-resolution magnetic resonance imaging accurate in predicting neurovascular compression in patients with trigeminal neuralgia? A singleblind study. Neurosurg Rev 2005;28:131-36

10. Zhou Q, Liu ZL, Qu CC, et al. Preoperative demonstration of neurovascular relationship in trigeminal neuralgia by using $3 \mathrm{D}$ FIESTA sequence. Magn Reson Imaging 2012;30:666-71 
11. Yamakami I, Kobayashi E, Hirai S, et al. Preoperative assessment of trigeminal neuralgia and hemifacial spasm using constructive interference in steady state-three-dimensional Fourier transformation magnetic resonance imaging. Neurol Med Chir (Tokyo) 2000;40: 545-55; discussion 555-56

12. Yoshino N, Akimoto H, Yamada I, et al. Trigeminal neuralgia: evaluation of neuralgic manifestation and site of neurovascular compression with 3D CISS MR imaging and MR angiography. Radiology 2003;228:539-45

13. Tarnaris A, Renowden S, Coakham HB, et al. A comparison of magnetic resonance angiography and constructive interference in steady state-three-dimensional Fourier transformation magnetic resonance imaging in patients with hemifacial spasm. Br J Neurosurg 2007;21:375-81

14. Girard N, Poncet M, Caces F, et al. Three-dimensional MRI of hemifacial spasm with surgical correlation. Neuroradiology 1997;39: 46-51

15. Raslan AM, DeJesus R, Berk C, et al. Sensitivity of high-resolution three-dimensional magnetic resonance angiography and three-dimensional spoiled-gradient recalled imaging in the prediction of neurovascular compression in patients with hemifacial spasm. J Neurosurg 2009;111:733-36

16. Holley P, Bonafe A, Brunet E, et al. The contribution of "time-offlight" MRI-angiography in the study of neurovascular interactions (hemifacial spasm and trigeminal neuralgia). J Neuroradiol 1996;23:149-56

17. Chang JW, Chang JH, Park YG, et al. Microvascular decompression in trigeminal neuralgia: a correlation of three-dimensional timeof-flight magnetic resonance angiography and surgical findings. Stereotact Funct Neurosurg 2000;74:167-74

18. Leal PR, Hermier M, Froment JC, et al. Preoperative demonstration of the neurovascular compression characteristics with special emphasis on the degree of compression, using high-resolution mag- netic resonance imaging: a prospective study, with comparison to surgical findings, in 100 consecutive patients who underwent microvascular decompression for trigeminal neuralgia. Acta Neurochir (Wien) 2010;152:817-25

19. Satoh T, Onoda K, Date I. Fusion imaging of three-dimensional magnetic resonance cisternograms and angiograms for the assessment of microvascular decompression in patients with hemifacial spasms. J Neurosurg 2007;106:82-89

20. Naraghi R, Tanrikulu L, Troescher-Weber R, et al. Classification of neurovascular compression in typical hemifacial spasm: three-dimensional visualization of the facial and the vestibulocochlear nerves. J Neurosurg 2007;107:1154-63

21. Satoh T, Omi M, Nabeshima M, et al. Severity analysis of neurovascular contact in patients with trigeminal neuralgia: assessment with the inner view of the 3D MR cisternogram and angiogram fusion imaging. AJNR Am J Neuroradiol 2009;30:603-07

22. González Sánchez JJ, Enseñat Nora J, Candela Canto S, et al. New stereoscopic virtual reality system application to cranial nerve microvascular decompression. Acta Neurochir (Wien) 2010;152: 355-60

23. Oishi M, Fukuda M, Hiraishi T, et al. Interactive virtual simulation using a $3 \mathrm{D}$ computer graphics model for microvascular decompression surgery. J Neurosurg 2012;117:555-65

24. Kondo A, Date I, Endo S, et al. A proposal for standardized analysis of the results of microvascular decompression for trigeminal neuralgia and hemifacial spasm. Acta Neurochirurgica (Wien) 2012; 154:773-78

25. Landis JR, Koch GG. The measurement of observer agreement for categorical data. Biometrics 1977;33:159-74

26. Morioka T, Hashiguchi K, Samura K, et al. Detailed anatomy of intracranial venous anomalies associated with atretic parietal cephaloceles revealed by high-resolution 3D-CISS and high-field T2weighted reversed MR images. Childs Nerv Syst 2009;25:309-15 